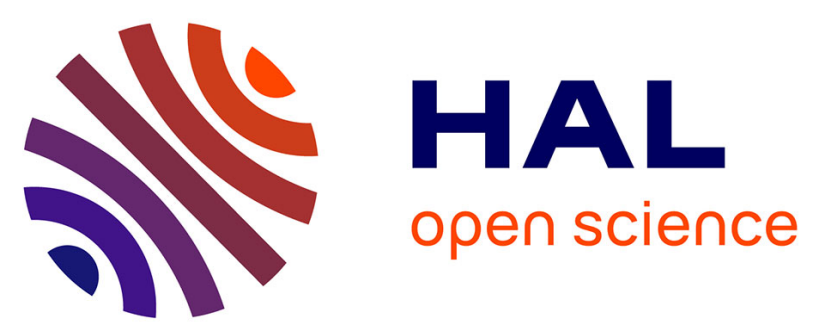

\title{
MAGNETIC FIELD DEPENDENCE OF THE ULTRASONIC ATTENUATION AND RESISTANCE OF A SUPERCONDUCTING GRANULAR LEAD FILM
}

\author{
Julien Schmidt, A. Schenstrom, M. Levy
}

\section{To cite this version:}

Julien Schmidt, A. Schenstrom, M. Levy. MAGNETIC FIELD DEPENDENCE OF THE ULTRASONIC ATTENUATION AND RESISTANCE OF A SUPERCONDUCTING GRANULAR LEAD FILM. Journal de Physique Colloques, 1985, 46 (C10), pp.C10-711-C10-714. 10.1051/jphyscol:198510156 . jpa-00225363

\section{HAL Id: jpa-00225363 https://hal.science/jpa-00225363}

Submitted on 1 Jan 1985

HAL is a multi-disciplinary open access archive for the deposit and dissemination of scientific research documents, whether they are published or not. The documents may come from teaching and research institutions in France or abroad, or from public or private research centers.
L'archive ouverte pluridisciplinaire HAL, est destinée au dépôt et à la diffusion de documents scientifiques de niveau recherche, publiés ou non, émanant des établissements d'enseignement et de recherche français ou étrangers, des laboratoires publics ou privés. 
JOURNAL DE PHYSIQUE

Colloque C10, supplément au $\mathrm{n}^{\circ} 12$, Tome 46, décembre 1985 page Cl0-711

MAGNETIC FIELD DEPENDENCE OF THE ULTRASONIC ATTENUATION AND RESISTANCE OF A SUPERCONDUCTING GRANULAR LEAD FILM*

\author{
J. SCHMIDT, A. SCHENSTROM AND M. LEVY \\ Department of Physics, University of Wisconsin-Milwaukee, \\ Milwaukee, WI 53201, U.S.A.
}

\begin{abstract}
We report on the measurements of the ultrasonic attenuation and the d.c. resistance of a superconducting granular lead $f i l m$ as a function of both temperature and applied magnetic field. In the absence of an applied magnetic field, the d.c. resistance and ultrasonic attenuation can be accounted for, qualitatively, by using percolation theory. When a magnetic field is applied, we find that if we assume that both the ultrasonic attenuation and the resistivity are proportional to the effective area through which the field penetrates the film, we can deduce magnetization curves for the film from the normalized attenuation and resistance data. The shapes of these magnetization curves are consistent with those of a type II superconductor.
\end{abstract}

In this paper will report on the measurements of the d.c. resistance and surface acoustic wave (SAW) attenuation of a superconducting granular lead $\mathrm{film}$ as a function of both temperature and magnetic field. The film that was studied was reactively diode R.F. sputtered from a $99.999 \%$ pure lead target in an argon/ oxygen atmosphere onto a $\mathrm{yz}-\mathrm{LiNbO}_{3}$ substrate. Prior to the sputtering, the substrate was equipped with a pair of interdigital electrodes for sending and receiving the surface acoustic waves, and with four platinum resistance contacts which were used to measure the resistance by a four-probe technique. The film is approximately $500 \AA$ thick and has a normal state sheet resistance of $1000 \Omega / 0$. Electron microscope photographs $/ /$ / show that a granular lead film with a normal state sheet resistance in this range consist of metallic granules with an average size of $500 \AA$ surrounded by oxide. The frequency of the surface waves used in this investigation was $700 \mathrm{MHz}$ and the SAW attenuation in the normal state was found to be $4.2 \mathrm{~dB} / \mathrm{cm}$.

\footnotetext{
+ Research supported by the Air Force Office of Scientific Research under grant $n^{\circ}$ AFOSR $84-0350$
} 
The mechanism producing the SAW attenuation is piezoelectric coupling of the surface wave to the film resistivity and not electron-phonon interaction $/ 2 /$. In the absence of an external magnetic field, the observed attenuation can be accounted for, qualitatively, by using a model based on percolation arguments (Kuper, Schmidt, Levy, Ron, Revzen and Shapiro, to be pub7ished). In this mode7, long-range superconducting order sets in by means of percolation between neighboring metallic grains. The granular $\mathrm{film}$ is treated as a random resistor network in which the resistors of the network represent the oxide barriers between neighboring metalific grains. The normal resistance of a particular junction-resistor is assumed to be of the form $R=R_{0} e^{\xi}$ where $R_{0}$ is some resistance and $\xi$ is a random variable distributed according to a distribution function $W(\xi)$. Thus, the values of the resistors in the network in the normal state will be distributed according to a function $W(R)$ which is derivable from $W(\xi)$. As the temperature is lowered below the transition temperature of the grains $T_{g}$, those grains whose Josephson coupling energy is greater than the available thermal energy will couple by means of Josephson tunneling. In the resistor network analogy, this corresponds to shorting out some of the resistors in the network. As the temperature decreases, the number of shorted junction-resistors increases. When the fraction of shorted junction-resistors reaches the percolation threshold, corresponding to a temperature $T_{C}$, an infinite superconducting cluster is formed in the $f_{i l m}$ and the d.c. resistance drops to zero and remains zero for $T<T_{c}$.

The ultrasonic attenuation, on the other hand, remains nonzero for $T<T_{c}$ because the surface wave effectively measures the resistance of "sub-networks" whose dimensions are on the order of the acoustic wavelength. When macroscopic superconductivity first occurs in the film (as determined by the d.c. resistance measurement), there are still regions of the film which contain junction-resistors that have not yet been shorted. These regions will prodice an attenuation so the SAW attenuation will decrease more slowly than the d.c. resistance, remaining nonzero even when the d.c. resistance is equal to zero.

Figure 1 shows the data obtained at $T=4.2 \mathrm{~K}$ when a magnetic field is applied perpendicular to the surface of the film. As it can be seen, the sample exhibits a large value for the upper critical field (approximately 60 kilogauss). The shapes of these curves can be accounted for, at least qualitatively, by using our random resistor network model. When a magnetic field is.applied, flux lines will be forced between the metallic grains and thus some of the superconducting junctions become normal. As the field is increased, more and more junctions will be made normal. For the d.c. resistance, there will be a value of the magnetic field for which the infinite superconducting cluster will be destroyed. This corresponds to the onset of resistance in Figure 1. Eventually, as the field is increased further, all of the junctions will be made normal and the d.c. resistance of the sample will equal its normal value.

The process is the same for the SAW attenuation -- as the magnetic field is increased, more and more junctions are forced into the normal state resulting in an increase in attenuation. Once a junction is made normal, there exists a region around the junction which is in the normal state. The magnetic field can be considered to penetrate the sample uniformily in this "effective" area containing the normal junction. Let us denote by $f$ the ratio of the total effective area associated with all of the normal junctions to the total area of the film. If we assume that the normalized attenuation is proportional to the effective area through which the magnetic field penetrates $/ 2 /$, then for a film containing a mixture of normal and superconducting regions we can write

$$
-4 \pi M=H-f H
$$

Since there is a residual attenuation at any temperature (compare figure 7 of reference 3 to our Figure 1), we have $f=\left(\alpha(H)-\alpha_{0}\right) /\left(\alpha_{n}-\alpha_{0}\right)$ where $\alpha_{n}$ is the normal state attenuation and $\alpha_{0}$ is the residual attenuation. Substituting this expression for $f$ into equation (1) we get 


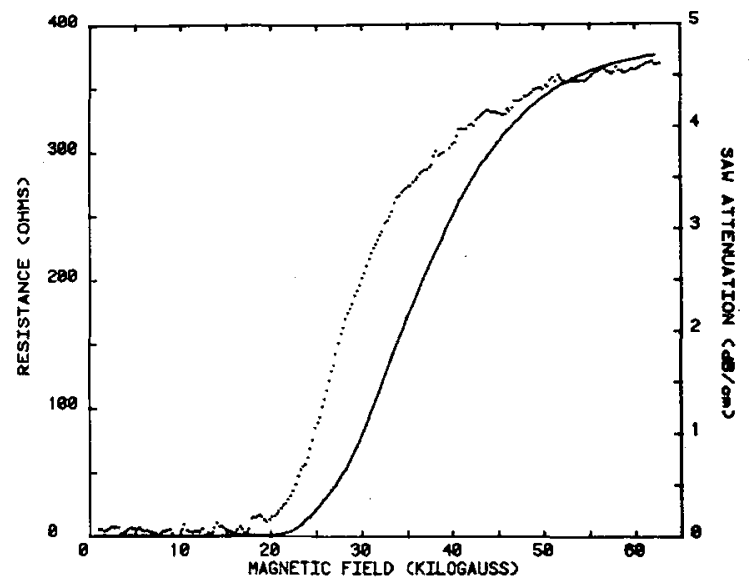

Fig. 1: Resistance (solid curve) and SAW attenuation (points) as a function of applied magnetic field for $T=4.2 \mathrm{~K}$.

$$
-4 \pi M=\left(\frac{\alpha_{n}-\alpha(H)}{\alpha_{n}-\alpha_{0}}\right) H
$$

If we plot the right hand of equation (2) as a function of $H$ using the normalized attenuation data, we get the curve shown in Figure 2 . It is interesting to note that this curve looks very much like the magnetization curve for a type II superconductor.

A similar analysis can be carried out for the resistance data. In this case, however, $f$ represents the fraction of the infinite cluster which is in the normal state, and not the fraction of the entire film that is normal. This is because the resistance measurement is only sensitive to the formation of the infinite superconducting cluster. For the resistance data we have $f=R(H) / R_{n}$ where $R_{n}$ is the normal state resistance. Substituting this expression for $f$ into equation (1), we get

$$
-4 \pi M=\left(\frac{R_{n}-R(H)}{R_{n}}\right) H
$$

Plotting the right hand of equation (3) as a function of $H$ using the resistance data, we get the second curve shown in Figure 2. Notice that this curve also looks very much like a magnetization curve for a type II superconductor. In addition, note that the maximum for the curve obtained from the resistance data occurs at a higher value of magnetic field then does the curve obtained from the SAW attenuation data. This is to be expected since the SAW attenuation is sensitive to all of the normal junctions in the $\mathrm{film}$ whereas the resistance is only sensitive to those normal junctions in the infinite cluster. 


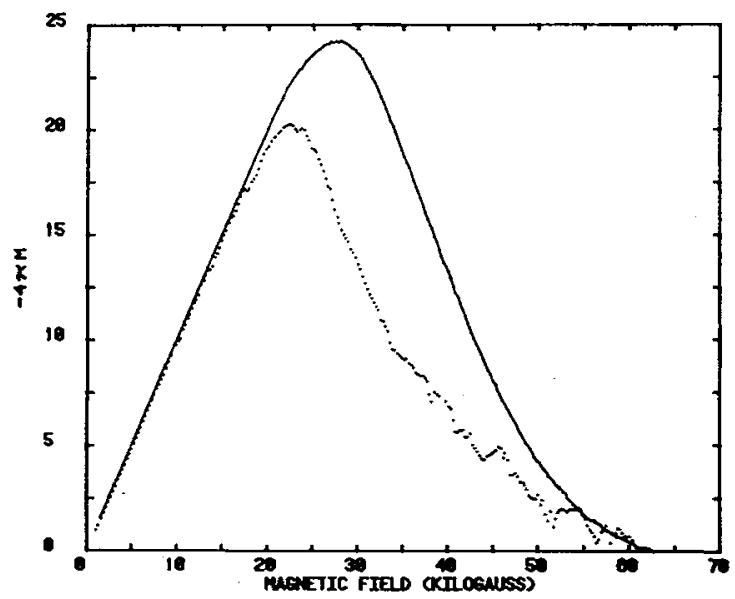

Fig. 2: Magnetization as a function of applied magnetic field from the SAW attenuation (points) and the d.c. resistance data (solid curve).

In conclusion, we have presented data on the d.c. resistance and SAW attenuation in a superconducting granular lead film as a function of both temperature and magnetic field. We have found that when a magnetic field is applied perpendicular to the surface of the $f i] \mathrm{m}$, the shapes of the resistance and attenuation curves could be explained, qualitatively, by using a model based on percolation in a random resistor network. Furthermore, by using simple arguments, we were able to deduce magnetization curves for the film (from the SAW attenuation data) and the infinite superconducting cluster (from the resistance data). These magnetization curves were found to be similar to that of a type II superconductor.

\section{References}

/1/ J. M. Vandenberg, S. Nakahara, and A. F. Hebard, J. Vac. Sci. Tech. 18, 268 (1981).

/2/ H. Tejima, J. Schmidt, C. Figura, and M. Levy, 1983 IEEE Ultrasonic Symposium Proceedings.

/3/ M. Levy, this conference. 